\title{
Cost-Effectiveness Analysis of Isavuconazole vs. Voriconazole as First-Line Treatment for Invasive Aspergillosis
}

\author{
Rachel Harrington - Edward Lee $\cdot$ Hongbo Yang $\cdot$ Jin Wei \\ Andrew Messali · Nkechi Azie $\cdot$ Eric Q. Wu · James Spalding
}

Received: August 23, 2016 / Published online: December 2, 2016

(C) The Author(s) 2016. This article is published with open access at Springerlink.com

\begin{abstract}
Introduction: Invasive aspergillosis (IA) is associated with a significant clinical and economic burden. The phase III SECURE trial demonstrated non-inferiority in clinical efficacy between isavuconazole and voriconazole. No studies have evaluated the cost-effectiveness of isavuconazole compared to voriconazole. The objective of this study was to evaluate the costs and cost-effectiveness of isavuconazole vs. voriconazole for the first-line treatment of IA from the US hospital perspective.
\end{abstract}

Methods: An economic model was developed to assess the costs and cost-effectiveness of

Enhanced content To view enhanced content for this article go to http://www.medengine.com/Redeem/ 0717F06062D4B7B0.

Electronic supplementary material The online version of this article (doi:10.1007/s12325-016-0443-1) contains supplementary material, which is available to authorized users.

R. Harrington $(\bowtie) \cdot$ E. Lee $\cdot$ N. Azie $\cdot$ J. Spalding Astellas Pharma Global Development, 1 Astellas Way, Northbrook, IL 60062, USA

e-mail: Rachel.Harrington@astellas.com

H. Yang · J. Wei · A. Messali · E. Q. Wu Analysis Group, Inc., 111 Huntington Ave, 14th Floor, Boston, MA 02199, USA isavuconazole vs. voriconazole in hospitalized patients with IA. The time horizon was the duration of hospitalization. Length of stay for the initial admission, incidence of readmission, clinical response, overall survival rates, and experience of adverse events (AEs) came from the SECURE trial. Unit costs were from the literature. Total costs per patient were estimated, composed of drug costs, costs of AEs, and costs of hospitalizations. Incremental costs per death avoided and per additional clinical responders were reported. Deterministic and probabilistic sensitivity analyses (DSA and PSA) were conducted.

Results: Base case analysis showed that isavuconazole was associated with a $\$ 7418$ lower total cost per patient than voriconazole. In both incremental costs per death avoided and incremental costs per additional clinical responder, isavuconazole dominated voriconazole. Results were robust in sensitivity analysis. Isavuconazole was cost saving and dominant vs. voriconazole in most DSA. In PSA, isavuconazole was cost saving in $80.2 \%$ of the simulations and cost-effective in $82.0 \%$ of the simulations at the $\$ 50,000$ willingness to pay threshold per additional outcome. 
Conclusion: Isavuconazole is a cost-effective option for the treatment of IA among hospitalized patients.

Funding: Astellas Pharma Global Development, Inc.

Keywords: Cost-effectiveness;

Invasive aspergillosis; Infectious diseases; Isavuconazole; Phase III trial; Sensitivity analyses; Voriconazole

\section{INTRODUCTION}

Invasive aspergillosis (IA) is a fungal infection that primarily affects immunocompromised individuals. In 2013, a total of 2990 hospitalizations with a primary diagnosis of IA, and 14,470 hospitalizations with either a primary or secondary diagnosis of IA, occurred in the USA [1]. Risk factors for IA include the presence of hematological malignancies, hematopoietic stem cell or solid organ transplant, severe and/or prolonged neutropenia, prolonged and/or high-dose immunosuppressive therapy, and chemotherapy [2]. The majority of cases of IA are encountered in patients with hematological malignancies and bone marrow transplant patients (43.0-68.0\%), with the remainder of cases occurring in solid organ transplant recipients (13.0-17.0\%) and other immunocompromised hosts (10.0-15.0\%) $[3,4]$.

IA is a serious, life-threatening condition. The reported 1-year overall survival rate following a diagnosis of IA among bone marrow transplant patients ranges from 10.0 to $40.0 \%$ in the literature $[5,6]$. Among critically ill patients admitted to an intensive care unit with proven IA, overall survival rates at 84 days after diagnosis of IA have been reported to be $21.0 \%$ [7]. A 2010 analysis of a national hospital administrative database found that the median length of hospitalization among patients diagnosed with IA was 18-26 days (depending on whether IA was a primary or secondary diagnosis) [8]. During this time, hospitals incurred a median cost between $\$ 32,465$ and $\$ 68,008$ (2006 USD), with antifungal medications accounting for $6.0-10.2 \%$ of those costs [8]. A 2009 analysis of data from the 2003 Nationwide Inpatient Sample concluded that the length of hospitalization for immunocompromised patients with IA was 1.8-12.4 times higher than the length of hospitalization of similarly high-risk immunocompromised patients without IA and the median hospital charges were 2.3-12.7 times higher (depending on diagnosis-related group) [9]. The annual total cost of IA in the USA, including hospitalizations, subsequent home healthcare, and outpatient medications, was estimated to be $\$ 674$ million in 1998 [10], the equivalent of $\$ 1.3$ billion today.

Current treatment guidelines, published before FDA approval of isavuconazonium sulfate, recommend several antifungal agents for the treatment of IA, including voriconazole, amphotericin B and its lipid formulations, itraconazole, posaconazole, and caspofungin $[11,12]$. Voriconazole, an antifungal triazole that inhibits fungal respiration and cell membrane function, is currently the preferred agent for first-line treatment of IA, both from a clinical [11] and an economic perspective $[13,14]$. Amphotericin B has a less favorable safety profile [15] and lower efficacy [15] compared to voriconazole, and it is recommended for patients who cannot be treated with voriconazole [11]. Other available agents offer limited efficacy benefits compared 
to voriconazole and therefore are recommended as salvage therapy agents after voriconazole or amphotericin B treatment [11]. A literature review conducted by Krueger and Nelson identified 10 pharmacoeconomic analyses in six different countries comparing voriconazole to other options for the treatment of IA, and all these analyses concluded that voriconazole was the most cost-effective therapy compared to other treatment options discussed above [13]. Another advantage of voriconazole is that it can be administered both orally and intravenously (IV). Voriconazole does present several possible disadvantages: possible toxicity of the IV formulation in patients with renal failure (due to required co-administration with solubility additive sulfobutylether- $\beta$-cyclodextrin) $[11,14]$, a twice daily dosing schedule of the oral medication because of relatively rapid metabolization [14], and a known risk of drug-drug interactions (due to its effect on the CYP2C19, CYP2C9, and CYP3A4 metabolic pathways) $[11,14]$.

Isavuconazonium sulfate, a novel triazole and prodrug of isavuconazole, received FDA approval for the treatment of IA and invasive mucormycosis in March 2015 [16]. The approval was granted on the basis of data from the SECURE clinical trial (NCT00412893), which showed that isavuconazole has comparable efficacy with voriconazole [17]. All-cause mortality at day 42 (the SECURE trial primary endpoint) was similar in both treatment groups $(18.6 \%$ in the isavuconazole-treated group and $20.2 \%$ in the voriconazole-treated group). Overall adverse event (AE) rates were also similar $(96.1 \%$ of isavuconazole-treated patients and $98.5 \%$ of voriconazole-treated patients experienced at least one treatment-emergent $\mathrm{AE}$ ) [17]. However, statistically significant differences were observed in eye disorders, hepatobiliary disorders, and skin disorders, with isavuconazole-treated patients having significantly lower rates of these events [17]. Similar to voriconazole, isavuconazole is also available in both oral and IV formulations. Its IV formulation, however, does not require solubility additive sulfobutylether- $\beta$-cyclodextrin, which has been associated with potential nephrotoxicity in the voriconazole IV formulation [18]. Oral isavuconazole has a longer half-life than oral voriconazole, allowing for once daily dosing. In addition, the metabolism of isavuconazole is limited to the CYP3A4 and CYP3A5 pathway [18].

In order to determine the optimal treatment option, it is important to thoroughly evaluate new therapies against existing standards by comparing efficacy, safety, costs, and cost-effectiveness. No existing study compares the costs and cost-effectiveness of isavuconazole vs. voriconazole. To fill this gap, the current study has been conducted to estimate the total cost per treated patient and evaluate the cost-effectiveness of isavuconazole vs. voriconazole from a US hospital perspective.

\section{METHODS}

\section{Model Overview, Perspective, and Population}

A cost-effectiveness decision tree model was developed in Microsoft Excel 2010 (Fig. 1). The target population included patients with proven, probable, or possible invasive fungal disease caused by the Aspergillus species or other filamentous fungi (reflecting the SECURE trial population) [17]. Two treatments, isavuconazole and voriconazole, were compared. The model estimated the total cost per IA patient treated with each product, with total cost defined as the sum of drug, AE, and hospital stay costs. Incremental cost per death avoided and the incremental cost per additional 


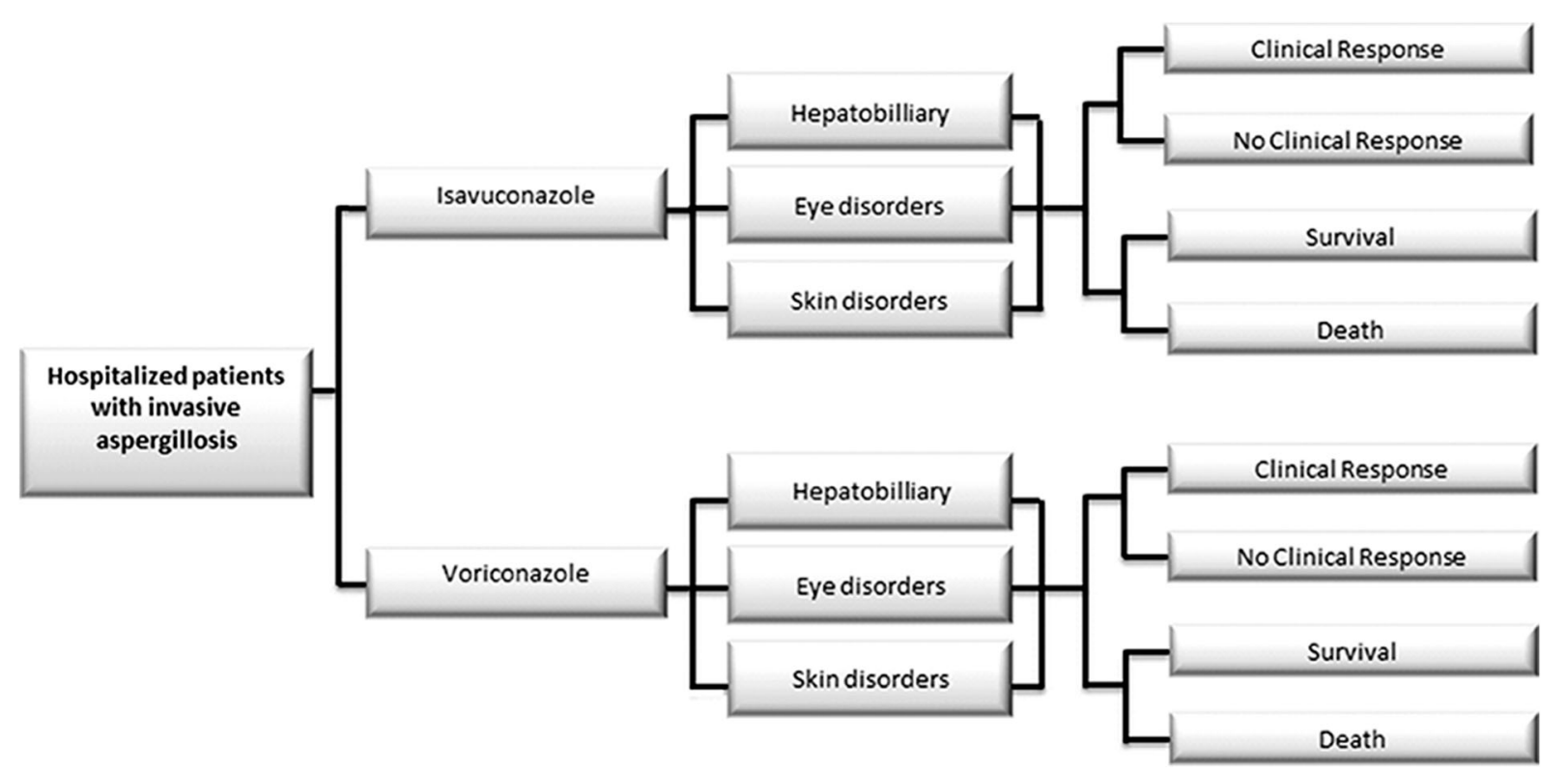

Fig. 1 Clinical progression of patients through the model. $I A$ invasive aspergillosis. Hospitalized patients with IA would enter the model and they could receive either isavuconazole or voriconazole. While receiving treatments, patients could experience adverse events related to

responder comparing isavuconazole to voriconazole were also estimated. The model was developed from a US hospital perspective, in which only direct costs incurred during the hospitalization were considered, with a time horizon of one hospital stay (including readmissions occurring within 30 days of the original discharge). Readmission within 30 days was considered given that it was a prespecified endpoint in the clinical trial. Because this time horizon is less than 1 year, discounting of costs and effectiveness measures was not necessary. This article does not contain any new studies with human or animal subjects performed by any of the authors. The model relied only on the summary statistics from the SECURE trial (ClinicalTrials.gov identifier NCT00412893) and patient level data was not used. Institutional review board (IRB) review was not needed. hepatobiliary, eye, and skin disorders. Patients were evaluated for survival and clinical response at the end of model cycle. Length of stay and likelihood of readmission (not depicted here) are also considered in the calculation of healthcare costs

\section{Efficacy and Safety Inputs}

Efficacy inputs, mortality and clinical response, for both treatment arms were extracted from the SECURE trial (Table 1) [17]. Mortality was the primary efficacy endpoint and clinical response was the secondary endpoint in the SECURE trial. As was previously described, all-cause mortality at day 42 was $18.6 \%$ for isavuconazole-treated patients and $20.2 \%$ for voriconazole-treated patients [adjusted difference $=-1.0 \%, 95 \%$ CI $(-7.8,5.7 \%)$ ] [17]. Clinical response rates were $62.0 \%$ and $60.3 \%$, respectively [adjusted difference $=0.4 \%, 95 \%$ CI $(-10.64,11.53 \%)]$ [17]. The differences in these two outcomes were not statistically significant. The median length of stay of the initial hospitalization was 13 days for isavuconazole-treated patients and 15 days for voriconazole-treated patients [19]. Following 
Table 1 Model inputs: efficacy, dosing schedule, and costs

\begin{tabular}{|c|c|c|c|}
\hline \multirow{2}{*}{$\begin{array}{l}\text { Definition } \\
\text { Efficacy }\end{array}$} & \multicolumn{2}{|c|}{ Base case value } & \multirow[t]{2}{*}{ Source/notes } \\
\hline & ISAV & VORI & \\
\hline Mortality rate & $18.6 \%$ & $20.2 \%$ & $\begin{array}{l}\text { SECURE trial [17]; measured at day } 42 \text { on the } \\
\text { basis of the intent to treat (ITT) population }\end{array}$ \\
\hline Clinical response rate & $62.0 \%$ & $60.3 \%$ & $\begin{array}{l}\text { SECURE trial }[17] \text {; measured at end of } \\
\text { treatment on the basis of the modified ITT } \\
\text { population }\end{array}$ \\
\hline Initial hospital length of stay, days & 13.0 days & 15.0 days & SECURE trial [19] \\
\hline 30-day readmission rate & $18.3 \%$ & $24.4 \%$ & SECURE trial [19] \\
\hline Readmission length of stay, days & 6.0 days & & $\begin{array}{l}\text { Estimated from an administrative database study } \\
\text { of hospitalized patients with IA [20] }\end{array}$ \\
\hline Dosing schedule & Dose & $\begin{array}{l}\text { Frequency } \\
\text { (per day) }\end{array}$ & \\
\hline ISAV & & & Product label $[16,22]$ \\
\hline Day 1: IV infusion & $372 \mathrm{mg}$ & 3 & \\
\hline Day 2: IV infusion & $372 \mathrm{mg}$ & 3 & \\
\hline \multicolumn{4}{|l|}{ Day 3 to end of treatment } \\
\hline IV infusion & $372 \mathrm{mg}$ & 1 & \\
\hline Oral (tablet) & $372 \mathrm{mg}$ & 1 & \\
\hline \multicolumn{4}{|l|}{ VORI } \\
\hline Day 1: IV infusion & $6 \mathrm{mg} / \mathrm{kg}$ & 2 & \\
\hline Day 2: IV infusion & $4 \mathrm{mg} / \mathrm{kg}$ & 2 & \\
\hline \multicolumn{4}{|l|}{ Day 3 to end of treatment } \\
\hline IV infusion & $4 \mathrm{mg} / \mathrm{kg}$ & 2 & \\
\hline Oral (tablet) & $200 \mathrm{mg}$ & 2 & \\
\hline $\begin{array}{l}\text { Treatment duration within } \\
\text { hospital, days }\end{array}$ & IV infusion & Oral & \\
\hline ISAV & 8.1 & 4.9 & SECURE clinical trial data [17] \\
\hline VORI & 8.9 & 6.1 & \\
\hline Drug cost (WAC) & \multicolumn{3}{|c|}{ Cost per unit } \\
\hline ISAV & & & ReadyPrice $^{\circledR}$ (Thomson) [23] \\
\hline IV infusion $(372 \mathrm{mg})$ & $\$ 238.50$ & & \\
\hline Oral (tablet) (186 mg) & $\$ 70.00$ & & \\
\hline \multicolumn{4}{|l|}{ VORI (generic) } \\
\hline IV infusion $(200 \mathrm{mg})$ & $\$ 122.07$ & & \\
\hline Oral (tablet) (200 mg) & $\$ 35.05$ & & \\
\hline
\end{tabular}

\begin{tabular}{|c|c|c|}
\hline Hospitalization $\cos \mathrm{t}^{\mathrm{a}}$ & & \\
\hline $\begin{array}{l}\text { Per diem cost of hospitalization } \\
\text { related to IA }\end{array}$ & $\$ 2898.31$ & HCUP NIS [1] \\
\hline
\end{tabular}

ISAV isavuconazole, $V O R I$ voriconazole, $I V$ intravenous, HCUP NIS Healthcare Cost and Utilization Project National Inpatient Sample

${ }^{\text {a }}$ Inflated to 2015 USD 
discharge from the initial hospitalization, $18.3 \%$ of isavuconazole-treated patients and $24.4 \%$ of voriconazole-treated patients had a readmission within 30 days [adjusted difference $=-6.0 \%, 95 \%$ CI $(-13.3,1.3 \%)]$. This difference was not statistically significant [19]. The median length of stay for readmissions (6 days) was obtained from an analysis of the Premier database of inpatients with a diagnosis of IA and who had a readmission [20]; in the absence of other information, it was assumed to be equal across treatment arms.

The model considered grade III and IV AEs whose overall incidence significantly differed between isavuconazole- and voriconazole-treated patients in the SECURE trial, i.e., eye disorders, hepatobiliary disorders, and skin disorders (individually described in ESM Appendix 1) [21].

\section{Dosing Frequency and Duration Inputs}

The dosing schedules for both voriconazole and isavuconazole were based on the doses administered in the SECURE trial [17], and they are consistent with their respective product labels [16, 22]. The current model assumed there was no vial wastage. Both isavuconazole and voriconazole were initiated as an IV infusion and could subsequently be converted to an oral formulation as early as day 3 , on the basis of physician evaluation. IV voriconazole was dosed on the basis of patients' weight, while administration of oral voriconazole, IV and oral isavuconazole was based on fixed dosing. The model assumed an average patient weight of $75 \mathrm{~kg}$ to estimate costs of IV voriconazole, which was the midpoint of the average weight of $82 \mathrm{~kg}$ of adults older than 20 years of age in the USA and the mean weight of $69 \mathrm{~kg}$ in the SECURE trial [21]. Details regarding the dosing of each treatment arm can be found in Table 1. The total duration of isavuconazole treatment (including IV and oral formulations) reported in the SECURE trial was 47.0 days on average, 8.1 days of which were IV treatment [17]. Only drugs administered within the hospital were considered; thus, the model included the costs of 8.1 days of IV and 4.9 days (13.0-8.1 days) of oral isavuconazole treatment. The total duration of voriconazole treatment was 46.4 days in the SECURE trial, 8.9 days of which were IV treatment [17]. The model considered voriconazole treatment as 8.9 days of IV and 6.1 days (15.0-8.9 days) of oral dosing.

\section{Cost Inputs}

Prices of isavuconazole and voriconazole were estimated on the basis of the wholesale acquisition costs (WACs), obtained from ReadyPrice $^{\circledR}$ (Thomson) (Table 1) [23]. Given that generic voriconazole is already available and with a lower price, the price of generic voriconazole was used. The price of $200 \mathrm{mg}$ of voriconazole was \$122.07 and \$35.05 for the IV and oral formulations, respectively. The price of $372 \mathrm{mg}$ of isavuconazonium sulfate (equivalent to $200 \mathrm{mg}$ of isavuconazole) was $\$ 238.50$ and $\$ 70.00$ for the IV and oral formulations, respectively.

The per diem cost of hospitalization due to IA was estimated at \$2898.31, using the 2013 HCUP Nationwide Inpatient Sample (NIS), a publicly available database created and maintained by the Agency for Healthcare Research and Quality (AHRQ) [1]. The same per diem cost of hospitalization was assumed for initial hospitalizations and readmissions (Table 1). All costs were inflated to 2015 USD [24].

The costs of managing AEs were estimated using published literature [25-37]. For acute AEs, the cost of managing the entire AE episode was included and this cost was assumed to be 
equal among the treatment arms. For AEs which could last beyond the initial hospitalization, the cost per $\mathrm{AE}$ reported in literature (typically reported as an annual cost) was prorated on the basis of the length of initial hospital stay (i.e., 13 days for isavuconazole and 15 days for voriconazole) for each treatment arm. Prorating AEs this way makes the assumption that those AEs begin on the first day of IA therapy. This assumption is used given the lack of detailed information on time of $\mathrm{AE}$ onset and the fact that costs of $\mathrm{AE}$ do not have a significant impact on the model's results. When no literature was available, the cost of each $\mathrm{AE}$ was assumed to be the cost of an inpatient consultation of moderate complexity. In the case of hepatobiliary disorders, the cost of a liver function panel was also included.

\section{Deterministic and Probabilistic Sensitivity Analyses (DSA and PSA)}

Deterministic sensitivity analysis (DSA) was carried out by increasing and decreasing one parameter at a time while maintaining the rest of the inputs at the base case value. Treatment duration, length of hospitalization (length of stay), readmission rate, per diem cost of hospitalization, drug costs, costs of $\mathrm{AE}$ treatment, clinical response rates, and mortality rates were all varied in the DSA to evaluate their impact. In addition, a sensitivity analysis assuming that a US payer perspective was included. This payer perspective included drug and $\mathrm{AE}$ costs throughout the total duration of isavuconazole and voriconazole treatment. The total duration was reported by Horn et al. (including inpatient and outpatient) [19]. Another sensitivity analysis assumed equal hospital length of stay, mortality, and clinical response rate among isavuconazole- and voriconazole-treated patients. For this analysis, the average of each input for isavuconazole and voriconazole was used for both treatments. Sensitivity analyses were also conducted by changing only the inputs of isavuconazole to make it less favorable than voriconazole. The parameter inputs were varied on the basis of either the confidence interval, if available, or a predefined percentage of the base case value (ESM Appendix 2).

Probabilistic sensitivity analysis (PSA) was carried out by assuming parametric distributions for each input parameter and resampling each parameter simultaneously from their respective distributions to re-estimate model outputs (ESM Appendix 2). Treatment duration was assumed to follow normal distributions, and mortality and clinical response rates were assumed to follow beta distributions. The standard errors of these parameters were estimated from the SECURE trial. The initial hospitalization length of stay follows a uniform distribution, with the min and max specified as $\mp 25.0 \%$ of the base case value. The per diem cost of hospitalization and the costs of managing AEs were assumed to follow gamma distributions, with standard errors assumed to be equal to one quarter of their respective means. Five-thousand random draws were conducted. PSA was conducted for all three outcomes: incremental cost per patient, incremental cost per death avoided, and incremental cost per clinical responder. For the incremental cost per patient, the results of the PSA were summarized in a cost-minimization acceptability curve based on incremental cost per patient. For the incremental cost per death avoided and the incremental cost per clinical responder, cost-effectiveness acceptability curves were plotted on the basis of net monetary benefit (NMB) values, which were estimated on the basis of willingness to pay threshold $\times$ incremental benefit - incremental costs. 
Table 2 Base case results: costs and cost-effectiveness of ISAV vs. VORI

\begin{tabular}{lll}
\hline Parameter & ISAV & VORI \\
\hline Total cost per patient & $\$ 44,748.38$ & $\$ 52,166.16$ \\
Total drug cost & $\$ 3571.85$ & $\$ 3869.99$ \\
$\mathrm{AE}$ cost & $\$ 317.42$ & $\$ 576.74$ \\
Initial hospitalization cost & $\$ 37,678.04$ & $\$ 43,474.66$ \\
Hospital readmission cost & $\$ 3181.07$ & $\$ 4244.77$ \\
Incremental cost per death avoided & ISAV is dominant over VORI \\
Incremental cost per additional clinical responder & ISAV is dominant over VORI & \\
\hline
\end{tabular}

$I S A V$ isavuconazole, $V O R I$ voriconazole, $A E$ adverse event

a "Dominant" indicates greater health benefits (survival, clinical response) and lower cost

\section{RESULTS}

The model estimated the total costs per treated patient receiving isavuconazole and voriconazole, the incremental cost per patient treated with isavuconazole vs. voriconazole, the incremental cost per death avoided (isavuconazole vs. voriconazole), and the incremental cost per clinical responder (isavuconazole vs. voriconazole).

Isavuconazole was associated with a lower total cost per patient compared to voriconazole $(\$ 44,748.38$ vs. $\$ 52,166.16)$. This was a result of lower drug costs ( $\$ 3571.85$ vs. $\$ 3869.99$ ), lower costs of AEs ( $\$ 317.42$ vs. $\$ 576.74$ ), lower costs of initial hospitalization $(\$ 37,678.04 \quad$ vs. $\$ 43,474.66)$, and lower costs of hospital readmissions (\$3181.07 vs. \$4244.77) among isavuconazole-treated patients than voriconazole-treated patients. Isavuconazole was dominant (lower costs and greater health benefits) against voriconazole with respect to incremental cost per death avoided and incremental cost per clinical responder (Table 2).

Isavuconazole was predicted to be associated with lower total cost per patient than voriconazole in each DSA except for when the initial hospitalization length was increased by $25.0 \%$ for isavuconazole-treated patients and left unchanged for voriconazole-treated patients (Table 3). All other DSAs estimated a lower cost for isavuconazole than voriconazole, ranging from $\$ 1411.05$ to $\$ 17,292.29$ savings per patient. The DSA using the US payer perspective (including both inpatient and outpatient drug and $\mathrm{AE}$ costs) estimated a cost saving of $\$ 4849.26$ for isavuconazole-treated patients, compared to voriconazole-treated patients. The DSA assuming equal LOS, mortality, and clinical response for isavuconazole and voriconazole estimated a cost saving of \$1411.05 for isavuconazole.

DSAs for incremental cost per death avoided and incremental cost per clinical responder showed isavuconazole was dominating against voriconazole in all but two cases: when the initial hospitalization length increased by $25.0 \%$ for isavuconazole-treated patients but remained unchanged for voriconazole-treated patients, and when the relevant efficacy outcome, i.e., 42-day mortality rate or clinical response rate, changed unfavorably for isavuconazole but remained unchanged for voriconazole (making isavuconazole worse than voriconazole). 
Table 3 Deterministic sensitivity analysis results, ISAV vs. VORI

\begin{tabular}{|c|c|c|c|c|c|c|}
\hline \multirow[t]{2}{*}{ Parameter } & \multicolumn{2}{|c|}{ Total cost per patient } & \multicolumn{2}{|c|}{$\begin{array}{l}\text { Incremental cost per } \\
\text { death avoided }\end{array}$} & \multicolumn{2}{|c|}{$\begin{array}{l}\text { Incremental cost per } \\
\text { clinical responder }\end{array}$} \\
\hline & $\begin{array}{l}\text { Low SA } \\
\text { input }\end{array}$ & $\begin{array}{l}\text { High SA } \\
\text { input }\end{array}$ & $\begin{array}{l}\text { Low SA } \\
\text { input }\end{array}$ & $\begin{array}{l}\text { High SA } \\
\text { input }\end{array}$ & $\begin{array}{l}\text { Low SA } \\
\text { input }\end{array}$ & $\begin{array}{l}\text { High SA } \\
\text { input }\end{array}$ \\
\hline \multicolumn{7}{|c|}{ Changing the inputs for both ISAV and VORI } \\
\hline Treatment duration (IV) & $-\$ 7173.91$ & $-\$ 7661.64$ & Dominant & Dominant & Dominant & Dominant \\
\hline Treatment duration (oral) & $-\$ 7703.00$ & $-\$ 7132.55$ & Dominant & Dominant & Dominant & Dominant \\
\hline Total treatment duration & $-\$ 7522.57$ & $-\$ 7312.98$ & Dominant & Dominant & Dominant & Dominant \\
\hline $\begin{array}{l}\text { Initial hospital length of stay } \\
( \pm 25.0 \%)\end{array}$ & $-\$ 6161.21$ & $-\$ 8674.34$ & Dominant & Dominant & Dominant & Dominant \\
\hline $\begin{array}{l}\text { Initial hospital length of stay (mean } \\
\text { values) }\end{array}$ & $-\$ 2200.00$ & & Dominant & & Dominant & \\
\hline Readmission length of stay & $-\$ 7151.85$ & $-\$ 7683.70$ & Dominant & Dominant & Dominant & Dominant \\
\hline Readmission rate & $-\$ 7327.91$ & $-\$ 7484.42$ & Dominant & Dominant & Dominant & Dominant \\
\hline Per diem cost of hospitalization & $-\$ 5702.70$ & $-\$ 9132.86$ & Dominant & Dominant & Dominant & Dominant \\
\hline Total cost of AEs & $-\$ 7352.95$ & $-\$ 7482.61$ & Dominant & Dominant & Dominant & Dominant \\
\hline Drug prices (IV and oral) & $-\$ 7343.24$ & $-\$ 7492.31$ & Dominant & Dominant & Dominant & Dominant \\
\hline Payer perspective & $-\$ 4833.26$ & & Dominant & & Dominant & \\
\hline $\begin{array}{l}\text { Equal initial length of stay, } \\
\text { mortality rate, and clinical response } \\
\text { rate }\end{array}$ & $-\$ 1411.05$ & & - & & - & \\
\hline Mortality rate & - & - & Dominant & Dominant & Dominant & Dominant \\
\hline Clinical response rate & - & - & Dominant & Dominant & Dominant & Dominant \\
\hline \multicolumn{7}{|l|}{ Changing the inputs for ISAV only } \\
\hline Initial hospital length of stay & $-\$ 17,295.94$ & $\$ 2460.39$ & Dominant & $\$ 158,459.35$ & Dominant & $\$ 143,398.88$ \\
\hline Readmission rate & $-\$ 8216.44$ & $-\$ 6477.45$ & Dominant & Dominant & Dominant & Dominant \\
\hline Mortality rate & - & - & Dominant & $\$ 198,126.42$ & - & - \\
\hline Clinical response rate & - & - & - & - & $\$ 106,054.92$ & Dominant \\
\hline
\end{tabular}

$I S A V$ isavuconazole, $V O R I$ voriconazole, $I V$ intravenous, $A E$ adverse event

In all PSA simulations of the total cost per patient, the incremental cost per patient (comparing isavuconazole vs. voriconazole) was less than $\$ 50,000$ (ESM Appendix 3). In $80.2 \%$ of those simulations, isavuconazole was associated with a lower total cost per patient than voriconazole. At a willingness to pay (WTP) of $\$ 50,000$ per death avoided, $82.4 \%$ of simulations found isavuconazole to be cost-effective compared to voriconazole on the basis of NMB values (ESM Appendix 3). Similar results were seen when the cost per clinical 
responder was considered. At a WTP of $\$ 50,000$ per clinical responder, $81.8 \%$ of simulations indicated isavuconazole to be cost-effective compared to voriconazole on the basis of $\mathrm{NMB}$ values (ESM Appendix 3).

\section{DISCUSSION}

The current standard of care for the treatment of IA is voriconazole. However, isavuconazole, a novel triazole, was recently introduced in the USA for the treatment of IA. An appealing alternative treatment option, isavuconazole has comparable efficacy as voriconazole and isavuconazole is well tolerated compared with voriconazole, with fewer drug-related adverse events. Findings from this economic evaluation show that isavuconazole is associated with lower costs per treated patient and is a cost-effective option compared to voriconazole for the treatment of IA. Isavuconazole dominates voriconazole in terms of incremental cost per additional death avoided and per additional clinical responder.

In our analyses, the difference in the cost of hospitalization was the main driver of the difference in total costs between isavuconazole and voriconazole, contributing to $92.0 \%$ of the difference in total cost. Isavuconazole-treated patients were associated with a shorter duration of initial stay and lower rate of readmission compared to voriconazole-treated patients, leading to a lower hospitalization cost. Isavuconazole's shorter duration of treatment and fewer doses per day from day 3 onward led to lower isavuconazole drug cost, compared to voriconazole, despite a higher drug cost per day. Isavuconazole's lower incidence rates of AEs also translated into additional cost saving. As a result, the use of isavuconazole may lead to a cost saving of $\$ 7417.78$ per patient compared to voriconazole.
The study results were robust. In most sensitivity analyses, isavuconazole was associated with lower cost and greater health benefits (dominating scenario) compared to voriconazole. Only in situations where the initial length of hospital stay, mortality rate, and clinical response rate were made wholly unfavorable for isavuconazole (while maintaining voriconazole inputs) was isavuconazole no longer associated with cost savings or dominance, compared to voriconazole. Given the fact that isavuconazole is associated with numerically shorter length of stay, lower mortality, and higher response rates compared to voriconazole in the SECURE trial, these worst-case scenarios seem highly unlikely [17]. Even when LOS, mortality rate, and clinical response rate were assumed to be equal, isavuconazole was still associated with a lower cost per patient owing to the lower rate of readmission and associated readmission costs. In about $80.0 \%$ of the PSA simulations, isavuconazole is less costly than voriconazole; in the majority of the simulations (approx. $82 \%$ of cases), isavuconazole is also more cost-effective than voriconazole at a WTP of $\$ 50,000$ per additional outcome.

No prior studies have compared the cost-effectiveness of isavuconazole vs. voriconazole in the treatment of IA; however, there are multiple studies comparing voriconazole to other IA treatments [13, 38]. Those studies have concluded that voriconazole is the preferred therapy for IA in terms of cost-effectiveness. The current study utilized a similar methodology as these prior studies and found that isavuconazole is associated with a lower cost per patient and dominates voriconazole in cost-effectiveness analysis for the treatment of IA in the hospital setting.

The results of this study demonstrate that isavuconazole is a valuable addition to the IA 
armamentarium. The efficacy of isavuconazole is similar to that of voriconazole. Isavuconazole, however, is associated with a lower total cost per patient and is cost-effective compared to voriconazole. In the USA, where the cost of healthcare is steadily increasing, cost-effectiveness analyses are increasingly important in helping healthcare decision-makers to evaluate different reimbursement decisions and in helping physicians and patients make the optimal selection of therapy for a given condition. Given that IA imposes a substantial economic and clinical burden on society $[8,9]$, optimizing the treatment of IA by evaluating the cost-effectiveness of alternative treatment options could help improve the care and reduce the overall healthcare spending. Evidence from the current study could support payers and physicians in the process of therapy selection for the treatment of IA by providing valuable information for effective decision-making.

\section{Limitations}

This economic evaluation is subject to several limitations. First, the model did not consider costs of treating any underlying conditions. The costs associated with treating underlying diseases can be substantial and may vary greatly by disease condition. There is, however, no evidence that treatment with isavuconazole or voriconazole could impact the costs of underlying disease conditions. Second, in order to make an informed comparison of first-line treatment with isavuconazole vs. voriconazole, no subsequent therapy was considered. The results of the SECURE trial indicated that the clinical response rate was numerically higher (not statistically significant) with isavuconazole than with voriconazole treatment; therefore, not including subsequent treatment is expected to be conservative against the isavuconazole arm, where a lower proportion of patients might receive subsequent therapy and incur associated costs. Additionally, although clinical trials are the gold standard for determining the comparative efficacy of alternative treatments, they often have restrictive inclusion and exclusion criteria and thus patients in the trial may lack the heterogeneity presented in real-world clinical practice. Finally, as the perspective of this study was that of a US hospital (and US payer, in sensitivity analysis), our results may not be generalizable to other countries with different healthcare systems, practices, and prices.

\section{CONCLUSIONS}

This economic evaluation shows that isavuconazole may be a cost-effective option compared to voriconazole for the treatment of IA in the hospital setting. Isavuconazole was associated with a lower total cost per patient and dominated voriconazole in terms of incremental costs per additional death avoided and cost per additional clinical responder.

\section{ACKNOWLEDGEMENTS}

Sponsorship, article processing charges, and the open access charge for this study were funded by Astellas Pharma Global Development, Inc. The study sponsor was involved in all stages of the study research and manuscript preparation, but all authors participated in the design of the study and contributed to manuscript development. All named authors meet the International 
Committee of Medical Journal Editors (ICMJE) criteria for authorship for this manuscript, take responsibility for the integrity of the work as a whole, and have given final approval for the version to be published. The model was developed by Analysis Group, Inc. and analyzed and interpreted in collaboration with all authors. Manuscript drafts were prepared by the authors with editorial assistance provided by Ana Bozas, PhD, a professional medical writer employed by Analysis Group, Inc. and funded by Astellas Pharma Global Development Inc. All authors had full access to all of the data in this study and take complete responsibility for the integrity of the data and accuracy of the data analysis. All the authors made the decision to submit the manuscript for publication.

Disclosures. Rachel Harrington is an employee of Astellas Pharma Global Development, Inc. Edward Lee is an employee of Astellas Pharma Global Development, Inc. James R Spalding is an employee of Astellas Pharma Global Development, Inc. Nkechi Azie was an employee of Astellas Pharma Global Development, Inc. at the time of the study. Hongbo Yang is an employee of Analysis Group Inc. Jin Wei is an employee of Analysis Group Inc. Andrew Messali is an employee of Analysis Group Inc. Erice $\mathrm{Wu}$ is an employee of Analysis Group Inc. Analysis Group Inc. received research funding from Astellas Pharma Global Development Inc.

Compliance with Ethics Guidelines. This article does not contain any new studies with human or animal subjects performed by any of the authors. The model relied only on the summary statistics from the SECURE trial (ClinicalTrials.gov identifier NCT00412893) and patient level data was not used.
Institutional review board (IRB) review was not needed.

Data Availability. Data sharing is not applicable to this article as no datasets were generated or analyzed during the current study. A synopsis of the current research was presented in poster format at the AMCP Nexus 2015 meeting, which took place in Orlando, Florida, during October 26-29, 2015.

Open Access. This article is distributed under the terms of the Creative Commons Attribution-NonCommercial 4.0 International License (http://creativecommons.org/licenses/ by-nc/4.0/), which permits any noncommercial use, distribution, and reproduction in any medium, provided you give appropriate credit to the original author(s) and the source, provide a link to the Creative Commons license, and indicate if changes were made.

\section{REFERENCES}

1. HCUPnet: National and regional estimates on hospital use for all patients from the HCUP National Inpatient Sample (NIS). 2013 [cited 2015 November 30, 2015]. Available from: http://hcupnet.ahrq.gov/.

2. Kousha M, Tadi R, Soubani AO. Pulmonary aspergillosis: a clinical review. Eur Respir Rev. 2011;20(121):156-74.

3. Lin SJ, Schranz J, Teutsch SM. Aspergillosis case-fatality rate: systematic review of the literature. Clin Infect Dis. 2001;32(3):358-66.

4. Perkhofer S, Lass-Florl C, Hell M, et al. The Nationwide Austrian Aspergillus Registry: a prospective data collection on epidemiology, therapy and outcome of invasive mould infections in immunocompromised and/or immunosuppressed patients. Int $\mathrm{J}$ Antimicrob Agents. 2010;36(6):531-6.

5. Marr KA, Carter RA, Crippa F, Wald A, Corey L. Epidemiology and outcome of mould infections in hematopoietic stem cell transplant recipients. Clin Infect Dis. 2002;34(7):909-17. 
6. Upton A, Kirby KA, Carpenter P, Boeckh M, Marr $\mathrm{KA}$. Invasive aspergillosis following hematopoietic cell transplantation: outcomes and prognostic factors associated with mortality. Clin Infect Dis. 2007;44(4):531-40.

7. Taccone FS, Van den Abeele AM, Bulpa P, et al. Epidemiology of invasive aspergillosis in critically ill patients: clinical presentation, underlying conditions, and outcomes. Crit Care. 2015;19:7.

8. Kim A, Nicolau DP, Kuti JL. Hospital costs and outcomes among intravenous antifungal therapies for patients with invasive aspergillosis in the United States. Mycoses. 2011;54(5):e301-12.

9. Tong KB, Lau CJ, Murtagh K, Layton AJ, Seifeldin R. The economic impact of aspergillosis: analysis of hospital expenditures across patient subgroups. Int J Infect Dis. 2009;13(1):24-36.

10. Wilson LS, Reyes CM, Stolpman M, Speckman J, Allen $\mathrm{K}$, Beney J. The direct cost and incidence of systemic fungal infections. Value Health. 2002;5(1):26-34.

11. Walsh TJ, Anaissie EJ, Denning DW, Herbrecht R, Kontoyiannis DP, Marr KA, et al. Treatment of aspergillosis: clinical practice guidelines of the Infectious Diseases Society of America. Clin Infect Dis. 2008;46(3):327-60.

12. Denning DW. Invasive Aspergillosis. Clin Infect Dis. 1998;26(4):781-803.

13. Krueger KP, Nelson AC. Economic considerations in the treatment of invasive aspergillosis: a review of voriconazole pharmacoeconomic studies. Clinicoecon Outcomes Res. 2009;1:35-43.

14. Mikulska M, Novelli A, Aversa F, et al. Voriconazole in clinical practice. J Chemother. 2012;24(6):311-27.

15. Herbrecht R, Denning DW, Patterson TF, et al. Voriconazole versus amphotericin B for primary therapy of invasive aspergillosis. $\mathrm{N}$ Engl J Med. 2002;347(6):408-15.

16. Astellas Pharma U.S. Inc. CRESEMBA ${ }^{\circledR}$ (isavuconazonium sulfate). 2015; 1-28]. Available from: http://www.accessdata.fda.gov/drugsatfda docs/label/2015/207500s001,207501s001lbl.pdf.

17. Maertens JA, Raad II, Marr KA, et al. Isavuconazole versus voriconazole for primary treatment of invasive mould disease caused by Aspergillus and other filamentous fungi (SECURE): a phase 3, randomised-controlled, non-inferiority trial. Lancet. 2016;387:760-9.

18. Thompson GR, Wiederhold NP. Isavuconazole: a comprehensive review of spectrum of activity of a new triazole. Mycopathologia. 2010;170(5):291-313.
19. Horn D, Goff D, Khandelwal N, et al. Hospital resource use of patients receiving isavuconazole vs voriconazole for invasive mold infections in the phase III SECURE trial. J Med Econ. 2016;19(7):728-34.

20. Data on file. Analysis using Premier data to estimate the length of stay for 30-day readmission among patients who were initially hospitalized with invasive aspergillus infection. Astellas Pharma U.S. Inc.

21. Data on file. SECURE trial Clinical Data. Astellas Pharma U.S. Inc.

22. Pfizer I. VFEND (voriconazole) Prescribing Information. 2015 [cited 2015 November 30]. Available from: http://labeling.pfizer.com/ ShowLabeling.aspx?id=618.

23. ReadyPrice. 2009 [cited 2016 April 11]. Available from: http://www.rubali.com/thomsonreuters/drug information/index.php?frame=ready_price.html.

24. Consumer Price Index-All Urban Consumers. US Medical Care Services [cited 2016 April 11]. Available from: http://data.bls.gov/cgi-bin/surveymost?cu.

25. Bickers DR, Lim HW, Margolis D, et al. The burden of skin diseases: 2004 a joint project of the American Academy of Dermatology Association and the Society for Investigative Dermatology. J Am Acad Dermatol. 2006;55(3):490-500.

26. Davis KL, Mitra D, Medjedovic J, Beam C, Rustgi V. Direct economic burden of chronic hepatitis $C$ virus in a United States managed care population. J Clin Gastroenterol. 2011;45(2):e17-24.

27. Delong LK, Culler SD, Saini SS, Beck LA, Chen SC. Annual direct and indirect health care costs of chronic idiopathic urticaria: a cost analysis of 50 nonimmunosuppressed patients. Arch Dermatol. 2008;144(1):35-9.

28. Gao X, Stephens JM, Carter JA, Haider S, Rustgi VK. Impact of adverse events on costs and quality of life in protease inhibitor-based combination therapy for hepatitis C. Expert Rev Pharmacoecon Outcomes Res. 2012;12(3):335-43.

29. Johner A, Raymakers A, Wiseman SM. Cost utility of early versus delayed laparoscopic cholecystectomy for acute cholecystitis. Surg Endosc. 2013;27(1):256-62.

30. Koberlein J, Beifus K, Schaffert C, Finger RP. The economic burden of visual impairment and blindness: a systematic review. BMJ Open. 2013;3(11):e003471.

31. Sandler RS, Everhart JE, Donowitz M, et al. The burden of selected digestive diseases in the United States. Gastroenterology. 2002;122(5):1500-11. 
32. Schackman BR, Haas DW, Becker JE, et al. Cost-effectiveness analysis of UGT1A1 genetic testing to inform antiretroviral prescribing in HIV disease. Antivir Ther. 2013;18(3):399-408.

33. Udeh BL, Schneider JE, Ohsfeldt RL. Cost effectiveness of a point-of-care test for adenoviral conjunctivitis. Am J Med Sci. 2008;336(3):254-64.

34. Xakellis GC, Frantz R. The cost of healing pressure ulcers across multiple health care settings. Adv Wound Care. 1996;9(6):18-22.

35. Yu J, Asche CV, Fairchild CJ. The economic burden of dry eye disease in the United States: a decision tree analysis. Cornea. 2011;30(4):379-87.
36. Yun HR, Bae SC. Cost-effectiveness analysis of NSAIDs, NSAIDs with concomitant therapy to prevent gastrointestinal toxicity, and COX-2 specific inhibitors in the treatment of rheumatoid arthritis. Rheumatol Int. 2005;25(1):9-14.

37. Shadick NA, Liang MH, Phillips CB, Fossel K, Kuntz KM. The cost-effectiveness of vaccination against Lyme disease. Arch Intern Med. 2001;161(4):554-61.

38. Al-Badriyeh D, Heng SC, Neoh CF, Slavin M, Stewart K, Kong DC. Pharmacoeconomics of voriconazole in the management of invasive fungal infections. Expert Rev Pharmacoecon Outcomes Res. 2010;10(6):623-36. 